\title{
CAPÍTULO XIII \\ EL CAPITAL INTELECTUAL EN ASOCIACIONES DE PRODUCTORES DEL SUR DE COLOMBIA
}

\section{Juan Manuel Andrade Navia.}

Magister en Pensamiento Estratégico y Prospectiva, Magister en Gerencia del Talento Humano, candidato a doctor en Agroindustria. Investigador Junior de Colciencias. Docente de tiempo completo de la Universidad de Cundinamarca, seccional Girardot, Colombia. Correo: Jmanuelandrade@ucundinamarca.edu.co ORCID ID. 0000-0001-9644-0040

\section{Andrea Marcela Gasca Perdomo.}

Especialista en Alta Gerencia y Magíster en Administración de Empresas. Asesoría, apoyo y acompañamiento a instituciones del sector público y docencia. Correo: andreitagasca@gmail.com

\section{Ancízar Perdomo Gutiérrez.}

Especialista en Alta Gerencia y Magister en Administración de Empresas, con diplomados en Procedimientos Administrativos, Desplazamiento Forzado, Derechos Humanos, Derechos y Políticas Públicas, Emprendimiento y Gestión de Proyectos para el Desarrollo y la Innovación. Correo: ancizarperdomog@gmail.com

\section{Darío Benavides Pava.}

Magister en Administración de Empresas. Líder del Grupo de Investigación "Los Acacios". Docente de tiempo completo de la Universidad de Cundinamarca, seccional Girardot, Colombia. Correo: dbenavidesp@mail.unicundi.edu.co

\section{Henry Sánchez Pimentel}

Administrador Empresas, Especialista en Alta Gerencia y Magister en Administración de Empresas. Docente de Investigación grupo YUMA. Corporación Universitaria Minuto de Dios, Neiva - Colombia. Correo: hsanchezpim@uniminuto.edu.co

\section{Resumen}

El presente artículo tuvo el objetivo de medir las capacidades empresariales de las asociaciones de productores del sur de Colombia, beneficiados con el Proyecto Apoyo de Alianzas Productivas que corresponde a la financiación por parte del gobierno nacional a los pequeños y medianos productores rurales para organizarlos en asociaciones y acceder a los mercados formales. La medición se realizó por medio de la percepción de los miembros y asociados frente al capital intelectual desarrollado en sus componentes de capital humano, estructural y relacional. El estudio fue de corte cuantitativo y de tipo descriptivo, en el que se utilizó la técnica de la encuesta a través de un instrumento tipo escala Likert aplicado a 280 miembros asociados de 20 organizaciones. Frente a los resultados se obtuvo organizaciones con un nivel de avance organizativo desarrollado que supera el $60 \%$ en los componentes de capital humano en elementos como el conocimiento adquirido por sus miembros, la pertinencia profesional, los procesos de mejoramiento y la antigüedad de la asociación, y en el capital estructural en aspectos como las normas y valores, las certificaciones y marcas, el fondo rotatorio y los métodos de trabajo al interior de la organización; sin embargo, el 
capital relacional aún no alcanza su desarrollo óptimo obteniendo puntajes inferiores a los 60 puntos, especialmente en aspectos como el nivel de satisfacción del cliente y los nuevos socios y clientes.

Palabras clave: Capital intelectual, capital humano, capital relacional, capital estructural, asociaciones productivas.

\title{
THE INTELLECTUAL CAPITAL IN PARTNERSHIPS OF PRODUCERS IN SOUTHERN COLOMBIA
}

\begin{abstract}
The objective of this article was to measure the entrepreneurial capacities of the associations of producers in southern Colombia, benefiting from the Productive Alliances Support Project, which corresponds to the financing by the national government to small and medium rural producers to organize them in associations and access formal markets. The measurement was made through the perception of members and associates against the intellectual capital developed in its components of human, structural and relational capital. The study was of a quantitative and descriptive type, in which the survey technique was used through a Likert-scale instrument applied to 280 associate members of 20 organizations. Faced with the results, organizations were obtained with a level of organizational advance developed that exceeds $60 \%$ in the components of human capital in elements such as the knowledge acquired by its members, the professional relevance, the processes of improvement and the antiquity of the association, and in structural capital in aspects such as standards and values, certifications and brands, the revolving fund and working methods within the organization. However, the relational capital has not yet reached its optimal development, obtaining scores below 60 points, especially in aspects such as the level of customer satisfaction and the new partners and customers.
\end{abstract}

Keywords: Intellectual capital, human capital, relational capital, structural capital, productive associations.

\section{Capítulo resultado de un proyecto de investigación culminado.}

Se deriva del proyecto de investigación: La medición del capital intelectual en asociaciones de productores del sur de Colombia, beneficiados con el Proyecto Apoyo de Alianzas Productivas.

\section{Introducción}

Los escasos progresos que ha experimentado el empresariado en el sur de Colombia a través de un poco más de un siglo, en comparación con otras regiones con altos indicadores de generación empresarial y económicos y mucho más jóvenes, hacen preciso prestar atención sobre las pocas experiencias exitosas que se tienen en el ámbito local. 
Al respecto, se hace poderosamente atractivo indagar desde la perspectiva de la gestión del conocimiento en su dimensión del capital intelectual, las prácticas que permitieron que las empresas más representativas del sector a nivel local, pero con alcance nacional, logran altos niveles de desempeño y reconocimiento en el ámbito nacional.

Naranjo, Rubio \& Salazar (2011) manifiestan que, en algunas regiones las organizaciones han empezado a reflexionar sobre el capital intelectual, pero no han identificado, de manera rigurosa y sistemática, el capital intelectual que poseen, razón por la cual sus directivos carecen de la información necesaria para gestionar adecuadamente sus activos intangibles, aspecto crucial en el entorno actual de los negocios.

Al respecto, algunas investigaciones en regiones periféricas de Colombia como las realizadas por Andrade, Ramírez, Mendoza \& Sánchez (2018) sugieren una relación entre la innovación y el capital intelectual en las organizaciones que potencialmente conlleva a experimentar altos niveles de competitividad en las organizaciones.

Por tanto, es preciso que los gerentes y empresarios comprendan que la clave de las ventajas competitivas gira en torno a la gestión del conocimiento como generadora de las primeras, que implícitamente consideran el capital intelectual que desarrollan las empresas, involucrando las prácticas del talento humano, sus soportes institucionales y relacionales. Lo anterior, especialmente en las asociaciones de productores que corresponden a las organizaciones insignias del sur del país dada su alto vocación agroindustrial.

Así, esta investigación tuvo el objetivo de medir las capacidades empresariales de las organizaciones asociativas en el sur de Colombia a través de la percepción de los miembros y asociados frente al capital intelectual desarrollado en sus componentes de capital humano, estructural y relacional. Específicamente las asociaciones de productores beneficiadas con el Proyecto de Apoyo a las Alianzas Productivas.

En ese entendido, el Proyecto de Apoyo a las Alianzas Productivas (PAAP) del Ministerio de Agricultura y Desarrollo Rural se creó con el objetivo de vincular a los pequeños y medianos productores rurales para organizarlos en los mercados formales, de una manera justa, para mejorar sus condiciones de vida mediante generación de ingresos para su núcleo familiar, fomentando la asociatividad como una forma de fortalecer las capacidades empresariales, que redunde en el desarrollo regional agropecuario (Ministerio de Agricultura y Desarrollo Rural, 2016).

Este Proyecto tuvo su origen en el año 2002 y hasta el año 2016, había financiado 62 Alianzas Productivas, con un incentivo modular por valor total de $\$ 17.778 .553 .000$. En resumen, se beneficiaron asociaciones (Ministerio de Agricultura y Desarrollo Rural, 2016).

Finalmente, debido a las grandes inversiones que ha tenido el Estado este tipo de proyectos estimula la labor de conocer el estado de desarrollo del capital intelectual de dichas organizaciones en el contexto marginal de país. 


\section{Fundamentación Teórica}

\section{Teoría de recursos y capacidades}

En un primer momento la teoría de recursos y capacidades se basó en la concepción de la empresa como un conjunto de recursos, preocupándose del estudio de los factores sobre los que se apoyan las ventajas competitivas, para poder justificar la formulación de determinada estrategia de una empresa. Así, de acuerdo con Fernández \& Suárez (1996, 1) cada empresa es heterogénea dado que, posee diferente dotación de recursos, producto de su historia, suerte y decisiones pasadas, sobre las que se puede sustentar una ventaja competitiva; 2) puede mantener esa heterogeneidad a lo largo del tiempo, es decir, la ventaja competitiva puede ser sostenible, lo que le proporcionara una renta a largo plazo (Castillo y Portela, 2013, p.1)

Desde sus orígenes la economista británica Penrose (1958), argumentó que toda organización "es un conjunto de recursos productivos, unos de naturaleza física o tangible y otros de naturaleza humana" y añade que "en el proceso de producción no son los recursos en sí sino los servicios que tales recursos rinden. Los servicios que rinden los recursos son una función del modo en que se emplean; el mismo recurso empleado para fines diferentes o de manera diferente y en combinación con tipos o cantidades diversas de otros recursos rinde servicios también diferentes..." lo fundamental no es la propiedad en sí, aunque como antes se anotaba puede ser factor limitante frente a la competencia, lo que permite generar ventajas, sino la forma como se utilizan los recursos (Pulido, 2016, p.3)

Otras miradas contemplan que esta teoría se concibe bajo los modelos de enfoque estratégico para el análisis y desarrollo de las organizaciones. La Teoría de Recursos y Capacidades (TRC) constituye uno de los principales modelos teóricos que guían la investigación que se realiza en gestión estratégica, y su utilización es creciente. Esta tendencia, que inicia en los años ochenta del siglo pasado, se explica por distintos factores, entre los que destacan: la aceptación, dentro de la comunidad académica asociada a la gestión estratégica, de argumentos de corte económico que se utilizan en este enfoque para explicar el comportamiento y desempeño de la empresa, y en particular, sus mecanismos de construcción y mantenimiento de la ventaja competitiva.

La TRC considera que la disposición de una ventaja competitiva expresa que la empresa ha alcanzado un nivel de desempeño superior al de sus competidores, situación que le permite obtener beneficios extraordinarios, aún en el largo plazo. En este sentido, construir y sostener una ventaja competitiva constituye la clave del éxito de la empresa y señala la eficiencia con que la empresa utiliza sus recursos y capacidades (Reinoso, Flores y Cardoza, 2017).

Bajo la lógica de visionar la organización como una serie de procesos para reconfigurar estructuras con énfasis en la innovación, se plantea que una organización que puede, de manera permanente, reconfigurar su estructura con base en su propia experiencia de innovación. Todo esto concentrándose en el diagnóstico, análisis, explotación y renovación de los recursos y capacidades que darán respuesta coherente y anticipada, de ser posible, al juego del mercado a fin de obtener una mejor rentabilidad para la firma con base en esta premisa (Pulido, 2016). 
Si bien estas interpretaciones indican una forma de concebir las capacidades y recursos de acuerdo a su teoría, es indispensable saber que las precisiones teóricas de estos elementos se inician con las contribuciones de Penrose (1959) y Andrews (1977). Para Penrose (1959), la empresa es contemplada como un conjunto de recursos productivos que determinan la dimensión que puede alcanzar. Sin embargo, los elementos que contribuyen a su crecimiento son los servicios que aquellos recursos proporcionan, o el uso que de ellos se hace. Continuador de estas ideas, Wernerfelt (1984) concibe a los recursos como todos aquellos activos tangibles e intangibles que se vinculan a la empresa de forma semipermanente, como ocurre con marcar, conocimiento tecnológico propio, maquinaria, procedimientos eficientes o capital.

De otra parte, Shumpeter (1942) el incitador de esta corriente, puede llegar a comprender mejor los procesos de cambio dinámico como procesos de creación destructora provocados por las empresas. Así, tomando como punto de partida los recursos que controlan y las capacidades que dominan, las empresas tratan de identificar oportunidades novedosas. La intención de la firma innovadora es explotarlas rentablemente, bajo la forma de nuevos productos o procesos, los cuales alteran en un grado determinado la situación anterior. Con este comportamiento se consigue la obtención de rentas económicas durante un periodo concreto, hasta que disminuyen por efecto del proceso de imitación desarrollado por los competidores (Castillo y Portela, 2013)

Ubicando la reflexión para comprender el contenido de las partes de la teoría de recursos y capacidades, se encuentra que por recursos se entiende tanto a los inicios o ingresos en las operaciones de la firma, como planta y equipos, propiedad intelectual, entre otros, como a las capacidades y competencias de la gente. Lo importante es reconocer que por sí solos no crean valor, sino por la interacción entre estos y las capacidades organizacionales, siempre y cuando se cumplan con las siguientes características: ser difíciles de imitar por la competencia, ser valiosos, raros y difíciles de sustituir (Barney, 1991) A su vez Grant (1991) define las capacidades como la habilidad de desempeñar una tarea o actividad que involucra patrones complejos de coordinación y cooperación entre la gente y los recursos, donde se pueden encontrar I+D, servicio al cliente de clase superior, etc. Esto indica que a través de su gestión se conseguirán llevar a cabo las actividades que se han propuesto.

\section{Capital Intelectual}

La dimensión capital intelectual, plantea algunas reflexiones alrededor de las restricciones de su concepto (Mention 2012). Su denominación genérica designa el valor del conjunto de activos intangibles de una organización; la valoración de estos activos es una actividad de la que se ha ocupado la contabilidad financiera. Sin embargo, dada la escasa apreciación de los factores intangibles en la actividad económica (especialmente la organizativa) sólo hasta épocas recientes, la técnica contable ha prestado suficiente atención a su valoración. Debido a ello, la información sobre estos activos ha adolecido de la base teórica necesaria para establecer criterios de medición y reconocimiento, lo que dificulta la obtención de una imagen fiel del verdadero valor de las empresas. Algunos de los modelos existentes limitan las discusiones del capital intelectual en ideas de significados de producción y no toman en consideración otro intangible no-intelectual, tales como valores y cultura organizacional. 
Los modelos descritos tienen en cuenta los activos intangibles siendo principalmente activos intelectuales o activos conocidos, es decir, aquellos que los psicólogos atribuyen a la parte izquierda del cerebro. Sin embargo, existen otros activos intangibles (como los valores, la cultura organizacional, el talento, la motivación y el compromiso de los empleados). Incluso si estos activos afectivos no pueden ser etiquetados como "intelectuales", son de gran importancia para el éxito de las empresas y las organizaciones (Naranjo, Rubio y Salazar, 2011).

La reflexión alrededor de los componentes de la teoría de recursos y capacidades, es esencial abordar los elementos constitutivos del capital intelectual, específicamente el capital humano, capital estructural y capital relacional (Edvinsson \& Malone, 1998; European Commission, 2006; Sveiby, 1997). El primer componente está vinculado a las personas y en la mayoría de los trabajos revisados se le denomina capital humano.

El segundo componente se refiere a los procesos, estructuras, tecnología e innovación y se le llama capital estructural, aunque en algunos trabajos se separa el aspecto tecnológico del organizativo (Brooking, 1996; CIC, 2003; Bueno, 2003; Chen, 2004). El último componente se refiere a la relación de la empresa, principalmente con el cliente, por lo cual se le denomina en la mayoría de los trabajos como capital cliente. Sin embargo, las relaciones de la empresa son un espectro más amplio pues también ocurren con otros agentes vinculados al negocio, como los proveedores, los aliados, el gobierno y la competencia (Sveiby, 1997; Euroforum, 1998; McElroy, 2002). De este modo, siguiendo la línea de trabajos y propuestas más recientes (Intellectus, 2003; Ordóñez de Pablos, 2004), se opta por el nombre de capital relacional (Naranjo et al., 2011, p.59).

\section{Capital Humano}

Dentro de los componentes del capital intelectual en las organizaciones, es indispensable abordar unos de los elementos que en su dimensión hacen referencia al capital humano. Ubicarlo en un contexto determinado, se valora como una situación de la organización ligada al modelo económico; se reconoce que es rentable para las empresas y en general, para el desarrollo económico de una sociedad, invertir en la capacitación y en la educación de los empleados. El nivel de educación asociado a la potenciación del talento humano es el fundamento del despliegue económico de países como Japón, Corea del Sur y China. Estos países basaron su transformación en el modelo económico del crecimiento basado en las exportaciones, aplicado en primer lugar por Japón y luego por los demás (Mejía, Bravo y Montoya, 2013).

Desde otra perspectiva, en el cuadro de mando integral de Kaplan \& Norton (1996), el capital humano está representado por la "perspectiva de aprendizaje y conocimiento", e incluye aspectos tales como capacidades, habilidades y motivación de los trabajadores. Brooking (1996) entiende por capital humano los "activos centrados en el individuo", refiriéndose a la pericia, creatividad, habilidad para resolver problemas, liderazgo y capacidad de gestión de los individuos y los grupos. Sveiby (1997) denomina al capital humano competencias, aludiendo a aspectos como educación, habilidades (destacando las sociales), experiencia y valores, mientras que, para Edvinsson \& Malone (1997), tal capital sería el enfoque humano, que contempla el conocimiento, la motivación y el liderazgo de las personas (Naranjo et al., 2011). En la investigación se adoptó la concepción del capital humano comprendida como la capacidad de gestión de los individuos y los grupos. 


\section{Capital Relacional}

Respecto al capital relacional, este se entiende como el valor que tienen las relaciones existentes entre la empresa y los diferentes agentes económicos y sociales con los que interacciona en el desarrollo de su actividad (Marr, Schiuma y Neely, 2004) además de las percepciones que estos tengan sobre la organización (Edvinsson y Malone, 1997). Entonces, el capital relacional se asume como el conjunto de relaciones económicas, políticas e institucionales desarrolladas por la institución con agentes externos como alumnos, padres de familia, empleadores, organizaciones no gubernamentales, gobierno y sociedad en general. También incluye la percepción que dichos agentes tienen de la institución, su imagen, reputación y confiabilidad (Machorro, Mercado, Cernas y Romero, 2016).

Kaplan \& Norton (1993) se refieren al capital relacional como "perspectiva de los clientes", e incluyen aspectos como la adquisición y retención de clientes. Saint Onge (1996), Bontis (1998) y Chen et al (2004) lo denominan "capital cliente", que incluye, el primero de ellos, la cobertura, penetración, lealtad y rentabilidad de los clientes. El segundo contempla las relaciones con clientes, proveedores, agentes de la industria y el gobierno, y el tercero refiere la capacidad de mercado, la intensidad de mercado y la lealtad de los clientes. En el modelo de Brooking (1996), el capital relacional es llamado "activos de mercado", entre los que se encuentran los canales de distribución, las marcas, las licencias y las franquicias. Sveiby (1997) le denomina "estructura externa" en la que están las relaciones con clientes y proveedores, las marcas y la reputación de la empresa. Por su parte, Edvinsson \& Malone (1997) consideran el "capital cliente" como parte del capital estructural (Naranjo et al., 2011). Para la presente investigación se tomó la definición amplia del capital relacional que integra las relaciones con clientes, proveedores, agentes de la industria y el gobierno, incluidos los canales de distribución, las marcas, las licencias y las franquicias.

\section{Capital Estructural}

El capital estructural es la "perspectiva de los procesos internos" del Cuadro de Mando Integral (Kaplan y Norton, 1996) que incluye aspectos como los procesos internos y de innovación. Sveiby (1997) lo denomina "estructura interna", aludiendo a la cultura, organización informal, redes internas y sistemas informáticos y administrativos. (Edvinsson y Malone, 1997), indican que dicho capital equivale al "enfoque de procesos" y contempla los procesos organizativos y las tecnologías de la información. Así, para Saint Onge (1996), incluye sistemas, estructura, estrategia y cultura organizativa. Para Edvinsson \& Malone (1997), está integrado por hardware, software, bases de datos, estructura, patentes, marcas y relaciones con los clientes, aspecto éste que, como se verá, es más propio del capital relacional.

Euroforum (1998) incluye la cultura, la filosofía, la estructura, los procesos y la propiedad intelectual de la empresa, mientras que, para Bontis (1998), son las rutinas organizativas, la estructura, la cultura y los sistemas de información. Por su parte, McElroy (2002) incluye infraestructura y cualquier otro tipo de soporte para que el capital humano desarrolle adecuadamente su trabajo. Chen et al. (2004) aluden a la cultura, estructura, aprendizaje organizativo, procesos y sistemas de información. Joia (2004) plantea dentro de sus componentes la cultura, la estructura, las operaciones, los procesos, las bases de datos, 
los códigos, los estilos de dirección y las redes internas (Naranjo et al., 2011). Al respecto, la investigación acogió el concepto de capital estructural referido como la estructura, estrategia y cultura organizativa, incluyendo aspectos como las operaciones, los procesos, las bases de datos, los códigos, los estilos de dirección y las redes internas, entre otros.

\section{Metodología}

\section{Diseño}

La investigación fue de enfoque cualitativo y de tipo descriptivo (Hernández, Fernández y Baptista, 2010) para medir a escala numérica para la recolección de información, considerando categorías y herramientas que permitan contrastar el conocimiento, por medio de los instrumentos definidos para la consecución de los objetivos.

\section{Recolección de información}

Las herramientas metodológicas aplicadas fue la encuesta tipo Likert. El instrumento elaborado para la recolección de información consta de un número total de 33 afirmaciones (ítems) distribuidas en cada una de las dimensiones que componen el capital intelectual. Estas se aplicaron conforme a la disponibilidad de los miembros de las organizaciones, cuyo propósito fue conocer la percepción que poseen en el desarrollo del capital intelectual en la organización.

\section{Población y muestra}

El estudio se desarrolló en el marco de las organizaciones del sector solidario, en las cuales se aplicaron 280 cuestionarios a los colaboradores y asociados de 20 organizaciones. Al respecto, las organizaciones pertenecen a diferentes sectores económicos. La muestra estuvo compuesta por el $60 \%$ de hombres y el $40 \%$ de mujeres de diferentes edades, con edades que oscilaron entre los 20 y 50 años (ver tabla 1).

Tabla 1. Análisis estadísticos: Medías, desviaciones estándar y varianzas

\begin{tabular}{cl}
\hline Ítems & Valor \\
\hline $\mathrm{N}$ & 280 \\
\hline Media & 3,66 \\
\hline Desviación estándar & 1,21 \\
\hline Varianza & 2,14 \\
\hline
\end{tabular}

Fuente: Elaboración propia (2019). 


\section{Análisis de datos}

La escala de calificación para cada componente se distribuye conforme al número de afirmaciones contenidas. El instrumento Likert tiene una calificación de 1 a 5, conforme 1 representa totalmente en desacuerdo y 5 totalmente de acuerdo. Así mismo, se establecieron 5 cinco niveles de desarrollo entre No iniciado, embrionario, en desarrollo, desarrollado y consolidado.

\section{Resultados}

A continuación, se presentan los resultados de la aplicación de la escala Likert para medir las percepciones, sobre capital humano, estructural y relacional, de los colaboradores de las organizaciones del sector solidario en una región marginal. El Alfa de Cronbach fue de 0.952 , lo que indica la fiabilidad del instrumento utilizado (ver Tabla 2).

Tabla 2. Estadísticas de fiabilidad

\begin{tabular}{ccc}
\hline Alfa de Cronbach & Alfa de Cronbach basada en elementos estandarizados & N de elementos \\
\hline 0,952 & 0,952 & 33 \\
\hline
\end{tabular}

Fuente: Elaboración propia (2019).

\section{Capital humano}

En la tabla 3 se aprecian los aspectos que se midieron en el componente del capital humano. De acuerdo a los resultados obtenidos por los elementos se infiere que el modelo se explica con cuatros aspectos sobresalientes en la calificación: el conocimiento adquirido por sus miembros $(64,5)$, la pertinencia profesional $(64,5)$, los procesos de mejoramiento $(61,3)$ y la antigüedad de la asociación $(61,3)$.

Tabla 3. Elementos de análisis del Capital Humano

\begin{tabular}{cc}
\hline Ítems & Calificación \\
\hline Nivel conocimiento adquirido por empleados y socios & 64,5 \\
\hline Pertinencia profesional & 64,5 \\
\hline Programas de formación y capacitación & 54,8 \\
\hline Experiencia de miembros y empleados & 51,6 \\
\hline Procesos de mejoramiento & 61,3 \\
\hline Antigüedad de la asociación & 61,3 \\
\hline Posicionamiento en el mercado & 58,1 \\
\hline Ventajas competitivas & 58,1 \\
\hline
\end{tabular}

Fuente: Elaboración propia (2019). 
Respecto al capital humano de las organizaciones analizadas, se evidencia que en un $51,6 \%$ se encuentra en el nivel desarrollado, es decir, que adquiere atributos en los componentes establecidos, mientras que un $48,4 \%$, se ubica en el nivel consolidado, puesto que despliega los ejes misionales con madurez organizativa (ver Figura 1).

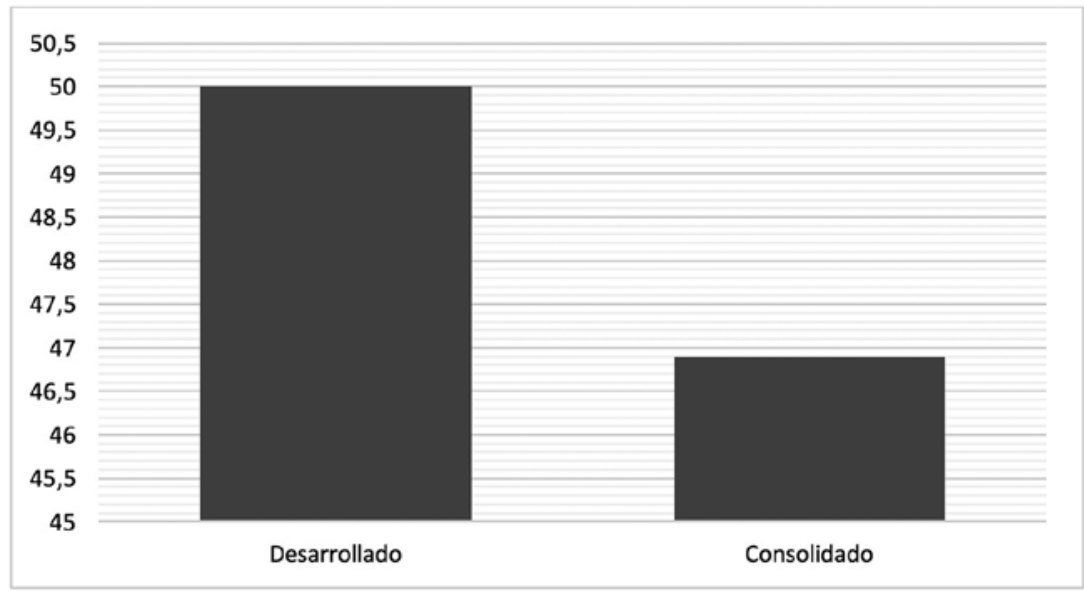

\section{Figura 1. Consolidado Capital Humano}

Fuente: Elaboración propia (2019).

\section{Capital estructural}

Frente a los resultados de la percepción obtenida por miembros y asociados con relación al capital estructural (ver Tabla 4), los aspectos con mayor relevancia en su calificación, hacen referencia a las normas y valores $(64,5)$, las certificaciones y marcas $(65,3)$, el fondo rotatorio $(62,7)$ y los métodos de trabajo al interior de la organización $(61,3)$. Sin embargo, existen aspectos críticos como el nivel de desarrollo tecnológico $(35,5)$.

Respecto a los resultados obtenidos del capital estructural, se infiere que el $61,3 \%$ se encuentra en un nivel desarrollado, es decir, que ejerce puntos fuertes en la organización; un $32,3 \%$ está consolidado o despliega los ejes misionales con madurez organizativa; y un $6,5 \%$ se encuentra en desarrollo o que adquiere atributos en los componentes establecidos (ver Figura 2). 
Tabla 4. Elementos de análisis del Capital Estructural

\begin{tabular}{cc}
\hline Ítems & Calificación \\
\hline Normas y valores & 64,5 \\
\hline Forma de trabajo & 61,3 \\
\hline Dirección y gerencia & 58,1 \\
\hline Formalización de procesos & 54,8 \\
\hline Nivel de conocimiento de estrategias y procesos & 41,9 \\
\hline Nivel de desarrollo tecnológico & 35,5 \\
\hline Procesos tecnológicos & 45,2 \\
\hline Información de socios & 54,8 \\
\hline Equipos tecnológicos e informáticos & 53,3 \\
\hline Certificaciones y marcas & 65,3 \\
\hline El diseño de productos y servicios & 56,0 \\
\hline Inversión en tecnología & 54,7 \\
\hline Desarrollo financiero & 50,7 \\
\hline Fondo rotatorio & 62,7 \\
\hline
\end{tabular}

Fuente: Elaboración propia (2019).

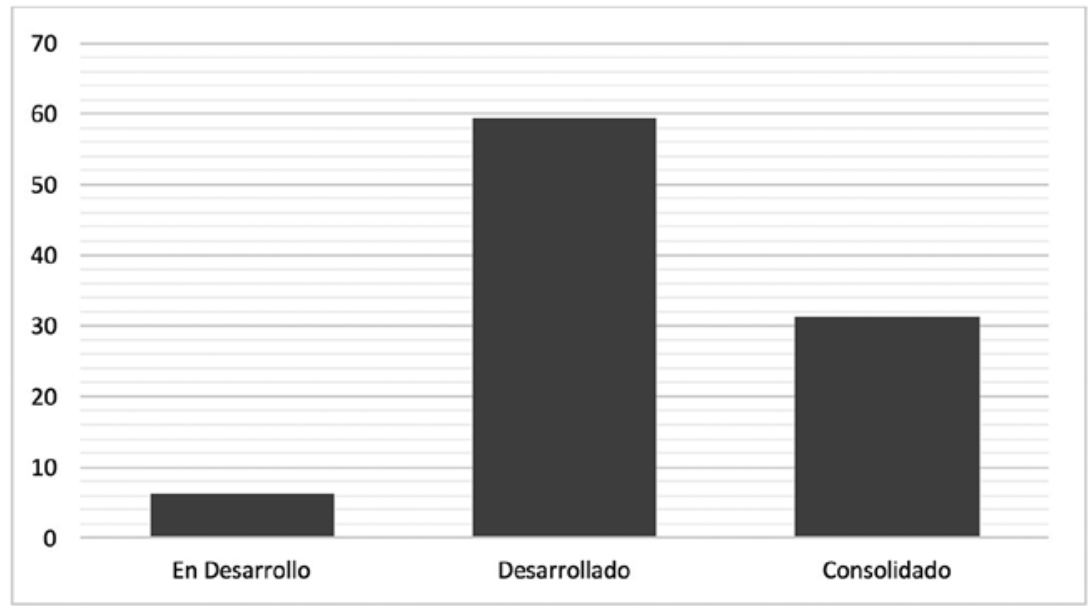

Figura 2. Consolidado Capital Estructural

Fuente: Elaboración propia (2019). 


\section{Capital relacional}

Los aspectos que toman mayor relevancia en la calificación del capital relacional, están soportados en elementos tales como el nivel de socios activos $(60,0)$, las alianzas y convenios $(56,0)$, la estructuración de estudios de mercado $(54,7)$ y la responsabilidad social de la organización $(54,7)$; sin embargo, se advierte que los puntajes obtenidos en este componente, no fueron superiores a los 60 puntos, lo cual difiere de los obtenidos en los componentes anteriores (ver Tabla 5).

Tabla 5. Elementos de análisis del Capital Relacional

\begin{tabular}{cc}
\hline Ítems & Calificación \\
\hline Nivel de clientes permanentes & 46,7 \\
\hline Nivel de socios activos & 60,0 \\
\hline Nivel de proveedores & 49,3 \\
\hline Nivel de satisfacción del cliente & 42,7 \\
\hline Nuevos socios y clientes & 44,0 \\
\hline Estructuración de estudios de mercado & 54,7 \\
\hline Inversión en mercadeo y publicidad & 52,0 \\
\hline Responsabilidad social y ambiental & 54,7 \\
\hline Alianzas y convenios & 56,0 \\
\hline Resolución de conflictos y problemáticas & 45,3 \\
\hline Imagen corporativa & 44,0 \\
\hline
\end{tabular}

Fuente: Elaboración propia (2019).

Los resultados obtenidos para el componente de capital relacional, hacen referencia en valorar un $54,84 \%$ en un nivel desarrollado, es decir, la organización ejerce puntos fuertes; un $25,81 \%$ en un nivel consolidado, despliega sus ejes misionales con madurez organizativa; y un 19,4 en un nivel en desarrollo, la cual implica adquirir tributos en los componentes establecidos (ver Figura 3). 


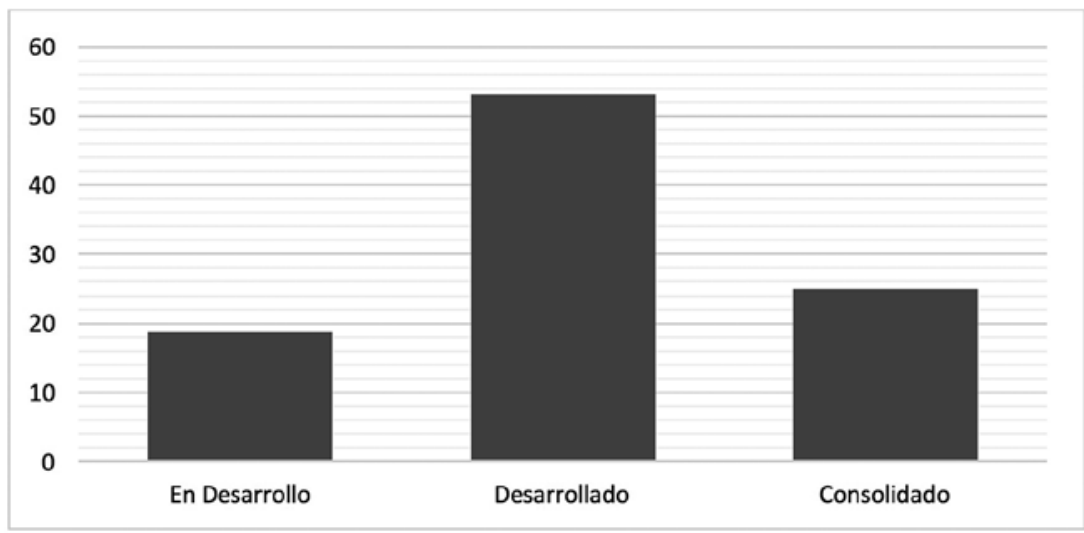

Figura 3. Consolidado Capital Relacional

Fuente: Elaboración propia (2019).

\section{Conclusiones}

Frente a los niveles de desarrollo del capital intelectual de las organizaciones analizadas reflejan una aceptabilidad y percepción positiva por parte de sus miembros y asociados. Con relación a los resultados, se pueden valorar las organizaciones con un nivel de avance organizativo desarrollado que supera el $60 \%$, es decir, que mantienen puntos fuertes en $\mathrm{su}$ estructura de funcionamiento, tanto interna como externamente, especialmente en los componentes de capital humano en elementos como el conocimiento adquirido por sus miembros, la pertinencia profesional, los procesos de mejoramiento y la antigüedad de la asociación, y en el capital estructural en aspectos como las normas y valores, las certificaciones y marcas, el fondo rotatorio y los métodos de trabajo al interior de la organización. Asimismo, se valora en más de un $40 \%$ de las organizaciones, como unas asociaciones que se encuentran en un nivel consolidada.

Sin embargo, se debe advertir que el componente relacional se encuentra en un estado incipiente, resaltando la necesidad de las organizaciones en fortalecer su componente de mercadeo y relaciones empresariales con el sector en el que se desenvuelven. Los aspectos identificados como más débiles fueron el nivel de satisfacción del cliente, la imagen corporativa y la gestión de nuevos socios y clientes.

\section{Referencias bibliograficas}

Andrade, J.M., Ramírez, E., Mendoza, J.A. y Sánchez, H. (2018). La innovación, el capital intelectual y la competitividad en las pequeñas empresas del sur colombiano. En: Tendencias en la investigación universitaria. Una visión desde Latinoamérica (Vol. 4), Fondo Editorial Universitario Servando Garcés. 
Andrews, K. (1977). The Concept of Corporate Strategic. Homewood, Illinois. Dow-Jones Irwin.

Barney, J.B. (1991). Firm resources and sustained competitive advantage. Journal of Management, 17, $99-120$.

Bontis, N. (1998). Intellectual Capital: An Exploratory Study that Develops Measures and Models. Management Decision, 36, 63-76.

Brooking, A. (1996). Intellectual Capital: Core Assets for the Third Millennium Enterprise. London: Thomson Business Press.

Castillo, I. y Portela, M. (2013). Tecnología y competitividad en la teoría de los recursos y capacidades. México.

CIC - Centro de Investigación sobre la Sociedad del Conocimiento (2003). Modelo Intellectus: medición y gestión del capital intelectual. $1^{\mathrm{a}} \mathrm{Ed}$. Madrid. Universidad Autónoma de Madrid.

Chen, J., Zhu, Z. y Yuan, H. (2004). Measuring intellectual capital: a new model and empirical study. Journal of Intellectual Capital, 5(1), $195-212$.

Edvinsson, L. y Malone, M.S. (1997). Intellectual capital: realizing y company's true value by finding its hidden brainpower. 1st Ed. Nueva York. Harper Collins Publishing.

Euroforum (1998). Medición del capital intelectual: modelo Intelect, I.U. Madrid. Euroforum Escorial.

European Commission (2006). RICARDIS (Reporting Intellectual Capital to augment research, development and innovation in SMEs). Report to the Commission of the High Level Expert Group on RICARDIS.

Fernández, J. y Suarez, I. (1996). La estrategia de la empresa desde una perspectiva basada en los recursos. Revista Europea de Dirección y Economía de la Empresa, 5(3): $73-92$.

Grant, R.M. (1991). The resource - based theory of competitive advantage: Implications for strategy formulation. California Management Review, Spring, 114 - 135.

Hernández, R., Fernández, C. y Baptista, M.P. (2010) Metodología de la Investigación (5 Ed.). México: McGraw Hill Educación.

Joia, L.A. (2004). Are frequent customer always a company`s intangible asset?: some findings drawn from an exploratory case study. Journal of Intellectual Capital, 5(4), $586-601$.

Kaplan, R.S. y Norton, D.P. (1993). The Balanced Scorecard. Massachusetts. Harvard Business School Press.

Kaplan, R.S. y Norton, D.P. (1996). Strategic learning \& the balanced scorecard. Strategy \& Leadership, 24(5), $18-24$. 
Machorro, F., Mercado, P., Cernas, D. y Romero, M. (2016). Influencia del capital relacional en el desempeño organizacional de las instituciones de educación superior tecnológica. INNOVAR, 26(60), 35 - 50.

Marr, B., Schiuma, G. y Neely, A. (2004). Intellectual capital - defining key performance indicators for organizational knowledge assets. Business Process Management Journal, 10(5), $551-569$.

McElroy, M.W. (2002). Social innovation capital. Journal of Intellectual Capital, 3(1), 30 - 39.

Mejia, A., Bravo, M. y Montoya, A. (2013). El factor del talento humano en las organizaciones. Ingeniería Industrial, 34(1), 2 - 11.

Ministerio de Agricultura y Desarrollo Rural (2016). Proyecto de Apoyo a las Alianzas Productivas. Bogotá D.C.

Naranjo, C., Salazar, L. y Rubio, J. (2011). El capital intelectual en Bellota-Colombia S.A. Tesis de maestría. Universidad Autónoma de Manizales, Manizales, Colombia.

Ordóñez de Pablos, P. (2004). Las cuentas de capital intelectual como complemento del informe anual. Economía Industrial, 357, 63-74.

Penrose, E. (1958). The theory of growth of the firm. Oxford.

Pulido, B. (2010). Teoría de los recursos y capacidades: el foco estratégico centrado en el interior de la organización. Sotavento M.B.A., 15, 54 - 61.

Reinoso, C., Flores, K. y Cardoza, L. (2017). La teoría de los recursos y capacidades: un análisis bibliométrico. Nova Scientia, 9(19), $411-440$.

Saint-Onge, H. (1996). Tacit knowledge: the key to strategic alignment of intellectual capital. Strategy and Leadership, 24(2), $10-16$.

Schumpeter, J.A. (1934). The Theory of Economic Development. Cambridge, MA. Harvard University Press.

Sveiby, K.E. (1997). The intangible assets monitor. Journal of Human Resource Costing and Accounting, 2(1), 73 - 97.

Wernerfelt, B. (1984). A resource - based view of the firm. Strategic Management Journal, $5,171-180$. 